Article

\title{
Effect of Thermal Vacancy on Thermodynamic Behaviors in BCC W Close to Melting Point: A Thermodynamic Study
}

\author{
Ying Tang ${ }^{1, *}$ and Lijun Zhang ${ }^{2, *}$ (D) \\ 1 School of Materials Science and Engineering, Hebei University of Technology, Tianjin 300130, China \\ 2 State Key Laboratory of Powder Metallurgy, Central South University, Changsha 410083, China \\ * Correspondence: ying_tang@hebut.edu.cn (Y.T.); lijun.zhang@csu.edu.cn (L.Z.)
}

Received: 28 July 2018; Accepted: 3 September 2018; Published: 7 September 2018

\begin{abstract}
As temperature increases, the thermal vacancy concentration in pure metals dramatically increases and causes some strongly non-linear thermodynamic behaviors in pure metals when close to their melting points. In this paper, we chose body-centered cubic (bcc) W as the target and presented a thermodynamic model to account for its Gibbs energy of pure bcc $\mathrm{W}$ from $0 \mathrm{~K}$ to melting point by including the contribution of thermal vacancy. A new formula for interaction part was proposed for describing the quadratic temperature behavior of vacancy formation energy. Based on the experimental/first-principles computed thermodynamic properties, all the parameters in the Gibbs energy function were assessed by following the proposed two-step optimization strategy. The thermodynamic behaviors, i.e., the strong nonlinear increase for temperature dependence of heat capacities at high temperatures and a nonlinear Arrhenius plot of vacancy concentration, in bcc W can be well reproduced by the obtained Gibbs energy. The successful description of thermal vacancy on such strongly non-linear thermodynamic behaviors in bcc $\mathrm{W}$ indicates that the presently proposed thermodynamic model and optimization strategy should be universal ones and are applicable to all other metals.
\end{abstract}

Keywords: thermal vacancy; thermodynamics; heat capacity; bcc tungsten

\section{Introduction}

Thermal vacancy is the simplest but extremely important structural defect in pure metals. As temperature increases, the thermal vacancy concentration in pure metals dramatically increases, and makes an apparent contribution to different physical quantities of materials, such as heat capacity, melting point, diffusivity, thermal conductivity, and so on [1-3]. Taking body cubic centered (bcc) W that has been proposed for use in the divertor of future fusion devices [4,5], for example, its thermal vacancy concentration can be larger than 0.02 at its melting point $[6,7]$. With such a large thermal vacancy concentration, the heat capacity of bcc $\mathrm{W}$ over the high-temperature region shows a strong non-linear increase, as demonstrated by most of the experimental data available in the literature [7-23] and plotted in Figure 1. Such strongly non-linear behavior on heat capacity in the region close to the melting point is related to the dramatic increase of thermal vacancy. The recent first-principles computed heat capacities of bcc W [24] are also superimposed in Figure 1 for a comparison with the experimental data. As clearly seen in Figure 1, the first-principles calculations only taking the harmonic/anharmonic vibration and electronic excitation into account [24] cannot accurately predict the heat capacity of $\mathrm{W}$ with such a non-linear increase over the high-temperature region. This fact indicates that the thermal vacancy contribution to heat capacity is noticeable at high temperatures. In addition to heat capacity, the thermal vacancy also shows obvious influence on self-diffusivity of bcc 
W. The Arrhenius plot for measured self-diffusivities in bcc W over a wide temperature range shows significant curvature [25]. Kraftmakher [3] pointed out that one probable reason for such curvature in self-diffusivities of bcc W lies in the fact that the concentration of thermal vacancy has a similar temperature dependence.

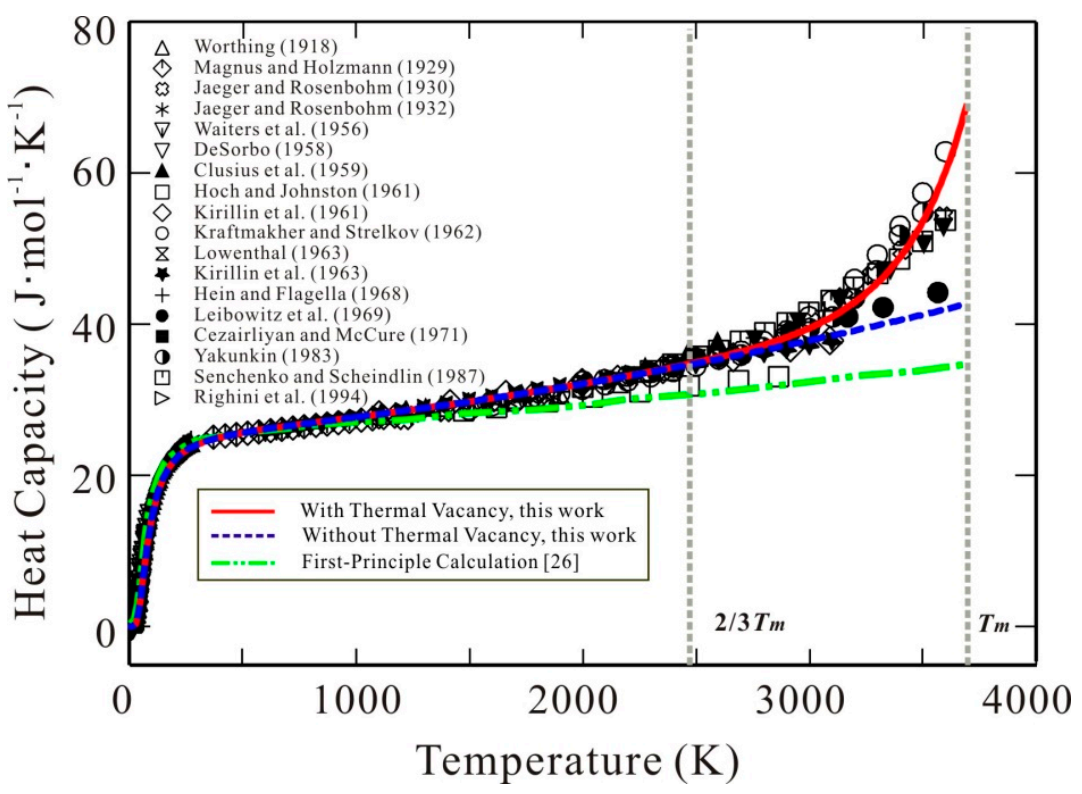

Figure 1. Heat capacity of body-centered cubic (bcc) W as a function of temperature. Symbols: Experimental data [7-23]. Solid line (red): Calculated results according to the presently established Gibbs energy for bcc W with thermal vacancy contribution. Dashed line (blue): Calculated results according to the presently established Gibbs energy for defect-free bcc W without thermal vacancy contribution. Dotted line (green): First-principles calculation [19], which does not include thermal vacancy contribution.

Furthermore, recent theoretical predictions $[26,27]$ show that formation entropy of vacancies is not constant as commonly assumed but increases with temperature, resulting in highly nonlinear temperature dependence in the formation energy. This point may naturally explain the strongly non-linear increase of the heat capacity at high temperatures and curvature in Arrhenius plot of vacancy concentration. Thus, in order to quantitatively describe the effect of thermal vacancy on these strongly non-linear thermodynamic behaviors in bcc $\mathrm{W}$, accurate prediction of the temperature-dependent thermal vacancy formation energy is the prerequisite, as is the major task of this paper.

\section{Thermodynamic Model for bcc W with Thermal Vacancy}

By considering the contribution of both thermal vacancy and $W$ atoms, the molar Gibbs free energy of pure element $\mathrm{W}$ in consistency with Compound Energy Formalism (CEF) can be described as [28,29]:

$$
G_{\mathrm{m}}=\frac{1}{y_{\mathrm{W}}}\left[y_{\mathrm{W}} G_{\mathrm{W}}+y_{\mathrm{va}} G_{\mathrm{va}}+R T\left(y_{\mathrm{W}} \ln y_{\mathrm{W}}+y_{\mathrm{va}} \ln y_{\mathrm{va}}\right)+y_{\mathrm{W}} y_{\mathrm{va}} \Omega\right]
$$

where $R$ is the gas constant, $T$ the absolute temperature, while $y_{\mathrm{w}}$ and $y_{\mathrm{va}}$ the site fractions of species $\mathrm{W}$ and thermal vacancy, respectively. The summation of $y_{\mathrm{w}}$ and $y_{\mathrm{va}}$ should be unity. $G_{\mathrm{W}}$ is the molar Gibbs energy of the defect-free element $W, G_{v a}$ the molar Gibbs energy of a virtual empty bcc lattice, while $\Omega$ is the interaction parameter. 


\subsection{Expression for $G_{W}$}

The molar Gibbs energy of the defect-free element $\mathrm{W}$, i.e., $G_{W}$, from $0 \mathrm{~K}$ to melting point in Equation (1) can be expressed by using the following physical model [30]:

$$
G_{\mathrm{W}}=E_{0}+\frac{3}{2} R \theta_{\mathrm{E}}+3 R T \ln \left[1-\exp \left(-\frac{\theta_{\mathrm{E}}}{T}\right)\right]-\frac{a}{2} T^{2}-\frac{b}{6} T^{3}-\int_{0}^{T}\left[\int_{0}^{T} \frac{C_{\mathrm{p}}^{\mathrm{mag}}}{T} d T\right] d T
$$

where $E_{0}$ is the total energy of ferromagnetic pure $\mathrm{W}$ at $0 \mathrm{~K}$ which can be directly obtained from the first principles calculations, while the second term is the energy of zero-point lattice vibration [31,32] with $\theta_{\mathrm{E}}$ as the Einstein temperature. The remaining four terms are derived from the integration of the heat capacity model for pure metals proposed in 1995's Ringberg Workshop [30]:

$$
C_{\mathrm{p}} \text { Pure } \mathrm{W}=3 R\left(\frac{\theta_{\mathrm{E}}}{T}\right)^{2} \frac{e^{\theta_{\mathrm{E}} / T}}{\left(e^{\theta_{\mathrm{E}} / T}-1\right)^{2}}+a T+b T^{2}+C_{\mathrm{p}}^{\mathrm{mag}}
$$

The first term in Equation (3) is the Einstein heat capacity, which is mostly contributed from harmonic vibration. The second term $a T$ in Equation (3) is related to electronic excitations and low-order anharmonic corrections, while the third term $b T^{2}$ contains the high-order anharmonic corrections. $C_{p}^{m a g}$ is the contribution from the magnetic ordering. It should be noted that, in order to obtain the heat capacities for defect-free $\mathrm{W}$, the coefficients $a$ and $b$ need to be evaluated by considering the experimental data without any contribution from thermal vacancy.

\subsection{Expression for $G_{v a}$}

For the molar Gibbs energy of a virtual empty bcc lattice, $G_{v a}$, in Equation (1), it is very difficult to define its standard reference value in a physical, meaningful way [33]. In order to make the balance between the different terms in Equation (1) over the wide temperature range, $G_{v a}$ is usually suggested to be proportional to temperature [27]. Besides, it would be desirable to set a general value for $G_{v a}$, which is independent of the elements, if for a universal usage for i.e., developing a common thermodynamic database. In 2014, Franke [28] pointed out that the value of $G_{v a}$ should be larger than a critical value (i.e., $(\ln 2-1 / 2) R T)$ to ensure a unique equilibrium state. In other words, the critical value can also ensure the second derivative of Gibbs energy $\partial^{2} G_{m} / \partial y_{v a}{ }^{2}$ to be positive at any temperatures, resulting in a unique equilibrium state. Moreover, such a critical value was obtained from a thorough mathematical analysis by Franke [28], and should thus serve as a common one which does not depend on the types of elements or phases.

In this work, the molar Gibbs energy of vacancy is simply set to $0.2 R T$, which is slightly larger than the critical value, as also proposed in Reference [28].

\subsection{Expression for Interaction Parameter $\Omega$}

As for the interaction parameter $\Omega$, one needs to figure out its relation with the non-linear concentration of thermal vacancy before proposing its expression. Based on Equation (1), for the equilibrium state of bcc $W$, one can have,

$$
\frac{\partial G_{\mathrm{m}}}{\partial y_{\mathrm{va}}}=\frac{1}{\left(1-y_{\mathrm{va}}\right)^{2}} G_{\mathrm{va}}+\frac{1}{\left(1-y_{\mathrm{va}}\right)^{2}} R T \ln y_{\mathrm{va}}+\Omega=0
$$

by applying the constraint $y_{w}+y_{v a}=1$. From Equation (4), the equilibrium concentration of thermal vacancy satisfies the following relation:

$$
y_{\mathrm{va}}=\exp \left(-\frac{G_{\mathrm{va}}+\Omega\left(1-y_{\mathrm{va}}\right)^{2}}{R T}\right)
$$


Meanwhile, the vacancy concentration can be also expressed as

$$
y_{\mathrm{va}}=\exp \left(-G_{\mathrm{va}}^{\mathrm{f}} / R T\right)=\exp \left(-\left(H_{\mathrm{f}}-T S_{\mathrm{f}}\right) / R T\right)
$$

where $G_{\mathrm{va}}^{\mathrm{f}}$ is the vacancy formation energy, $H_{\mathrm{f}}$ and $S_{\mathrm{f}}$ are the formation enthalpy and entropy of thermal vacancies, respectively. Comparing Equation (5) with Equation (6), it is easy to see that the value $G_{v a}+\Omega$ approaches to the formation energy of thermal vacancy in the limit of negligible vacancy concentrations. However, it seems to be very difficult and even impossible to separate the contributions of $G_{v a}$ and $\Omega$ from the formation energy of thermal vacancy [33]. The assumption of a constant entropy of formation of the thermal vacancy would introduce linear temperature dependence in $G_{v a}^{f}$ and hence lead to a constant prefactor to the Arrhenius plot of thermal vacancy concentration. However, for the Arrhenius plot of vacancy concentration with curvature behavior, i.e., $y_{\text {va }}$ in bcc $\mathrm{W}$, such assumption cannot give a reasonable description especially in the high temperature range. In order for an accurate thermodynamic description, the nonlinear temperature behavior of the vacancy formation energy needs to be considered. In a first approximation, we here assume a linear temperature dependence for $S_{\mathrm{f}}$; therefore, a temperature quadratic term will be included in $H_{\mathrm{f}}$ as well as in $G_{\mathrm{va}}^{\mathrm{f}}$ due to the thermodynamic relation $\left(\partial H_{\mathrm{f}} / \partial T\right)_{\mathrm{P}}=T\left(\partial S_{\mathrm{f}} / \partial T\right)_{\mathrm{P}}$. Moreover, we simply use the linear temperature dependence of $G_{v a}$ in the present work, i.e., 0.2RT, following the work of Franke [28]. In order to describe the quadratic temperature behavior of vacancy formation energy, we thus propose an expression for interaction parameter $\Omega$ in Equation (1):

$$
\Omega=A+B T+C T^{2}
$$

Here, $A, B$ and $C$ are the parameters, which can be assessed on the basis of the experimental data, like thermal vacancy concentration, and/or heat capacities at high temperatures. Substituting Equation (7) into Equation (5), one can easily see that the proposed interaction expression is able to describe the curvature between $\log y_{\text {va }}$ and $1 / T$.

Moreover, based on the molar Gibbs energy expression for bcc W together with Equations (4) and (5), its heat capacity can be derived as

$$
C_{\mathrm{p}}=C_{\mathrm{p}} \text { Pure } \mathrm{W}-y_{\mathrm{va}} T \frac{\partial^{2} \Omega}{\partial T^{2}}+\left(\Omega-T \frac{\partial \Omega}{\partial T}\right) \frac{\partial y_{\mathrm{va}}}{\partial T}
$$

in which $C_{\mathrm{p}}$ Pure $\mathrm{W}$ represents the heat capacity of defect-free element $\mathrm{W}$. The second and third terms on the right-hand side of Equation (8) denote the effects of thermal vacancy on heat capacity. When submitting Equation (7) into Equation (8), it can be found that the effects of thermal vacancy on heat capacity will become obvious with the increase of the temperature, which is excepted to describe the strongly non-linear behavior of thermodynamic properties near the melting point.

\section{Results and Discussion}

The heat capacity of bcc W was experimentally measured by several groups [7-23] from $0 \mathrm{~K}$ to melting point, as shown in Figure 1. The heat capacity shows a rapid increase at high temperatures especially close to the melting point. Moreover, the enthalpy increment of bcc W was also measured over a wide temperature range [14,34-39]. Additionally, the concentration of thermal vacancy in bcc W was also experimentally investigated at melting temperature [6,7]. Although no experimental vacancy concentration as a function of temperature was reported in the literature, Kraftmakher [3] derived the equilibrium vacancy concentrations in bcc W from the heat capacities with nonlinear increase [7].

The quantification of all the parameters in the molar Gibbs energy of bcc W (Equation (1)) can be divided into two steps. The first step is to fix the molar Gibbs energy of the defect-free element $\mathrm{W}$, i.e., $G_{W}$. Theoretically, the contribution of thermal vacancy should be excluded for the heat capacity and Gibbs energy of defect-free bcc W. However, it is also very difficult to separate the contribution of 
thermal vacancy for the experimental heat capacities completely in reality. The accurate first-principles calculations might provide the data for defect-free bcc W, but the current first-principles computed heat capacities of defect-free bcc W available in the literature [24] are lower than the experimental data above $1500 \mathrm{~K}$ (see Figure 1). As pointed out by Kraftmakher [3], the vacancy contribution to specific heat becomes visible only at temperatures above about two-thirds of the melting temperature (i.e., $2463 \mathrm{~K}$ for bcc W). Moreover, for bcc W, the magnetic effects on heat capacity can be ignored. In addition, Walford [40] experimentally measured the Debye temperature $\left(\theta_{\mathrm{D}}\right)$ of bcc $\mathrm{W}$, which was reported to be $377 \mathrm{~K}$. The corresponding Einstein temperature $\left(\theta_{\mathrm{E}}\right)$ can be evaluated as $269.2 \mathrm{~K}$ based on the relation $\theta_{\mathrm{E}} \approx 0.714 \theta_{\mathrm{D}}$ [41]. Then, the heat capacity of defect-free bcc $\mathrm{W}$ was obtained by fitting the experimental heat capacities and enthalpy increments below $2 / 3 T_{\mathrm{m}}$. Because there is no measured total energy ( $E_{0}$ in Equation (8)) of bcc $\mathrm{W}$ at $0 \mathrm{~K}$ in the literature, the first-principles calculated result due to Wang et al. [42] was directly used here to get the expression for Gibbs energy of defect-free bcc $\mathrm{W}$. The second step is to assess the three coefficients constituting the interaction parameter, $\Omega$, based on the experimental heat capacities over the range of $2 / 3 T_{\mathrm{m}} \sim T_{\mathrm{m}}$. The finally obtained thermodynamic parameters in Gibbs energy expression for bcc W are listed in Table 1.

Table 1. List of the evaluated thermodynamic parameters for bcc W.

\begin{tabular}{cl}
\hline Parameters & \multicolumn{1}{c}{ Values (Gibbs Energy in J/mol-atom; T in Kelvin) } \\
\hline$G_{\mathrm{W}}$ & $\begin{array}{l}E_{0}+\frac{3}{2} R \theta_{\mathrm{E}}+3 R T \ln \left(1-\exp \left(-\frac{\theta_{\mathrm{E}}}{\mathrm{T}}\right)\right)-1.085 \times 10^{-3} T^{2}-1.1835 \times 10^{-7} T^{3} \\
\left(E_{0}=-1228665.43, \quad \theta_{\mathrm{E}}=269.2\right)\end{array}$ \\
\hline$G_{\mathrm{Va}}$ & $+0.2 R T$ \\
\hline$\Omega$ & $+229615.89+12.73 T-1.1274 \times 10^{-2} T^{2}$ \\
\hline
\end{tabular}

Figure 1 shows the calculated heat capacity for bcc W using the presently obtained Gibbs energy expression in comparison with the experimental data [7-23]. As can be seen in the figure, excellent agreement between the calculations and the experiments is obtained. For a comparison, the calculated heat capacity of defect-free bcc $W$ is also superimposed in Figure 1 . The deviation between the heat capacity with and without thermal vacancy is quite obvious at high temperatures, and can reach $26.5 \mathrm{~J}$ $(\mathrm{mol} \mathrm{K})^{-1}$ at melting temperature. It indicates that the thermal vacancy has a significant effect on the thermodynamic properties of the pure metals. Moreover, one can clearly see the strongly non-linear behavior of heat capacities of bcc W, i.e., the dramatic increase of heat capacity at high temperatures close to melting point can be well reproduced by the presently obtained Gibbs energy of bcc W with thermal vacancy.

Figure 2 displays the presently calculated heat contents $\left(\mathrm{H}-\mathrm{H}_{298}\right)$ of bcc $\mathrm{W}$ with and without thermal vacancy along with experimental data [14,34-39]. As shown in Figure 2, the calculated results with thermal vacancy reproduce the reported data very well, while the results without thermal vacancy show a small deviation from the experimental data at the high temperature range. It indicates that the present thermodynamic description can well describe the thermal vacancy contribution to the heat contents, though the thermal vacancy contribution to heat content at high temperatures is relatively small compared with that to heat capacity.

Figure 3 presents the model-predicted 10-base logarithm values of thermal vacancy concentration of bcc W as a function of 10,000/T due to the obtained Gibbs energy of bcc W with thermal vacancy, compared with the experimental data at melting temperature [6,7]. As can be seen in the figure, the model-predicted thermal vacancy concentration of bcc W is 0.018 , which agrees well with the experimental data [6,7]. Moreover, as expected, such Arrhenius plot of vacancy concentration shows a clear curvature, which well reproduces the non-linear behavior stated by Kraftmakher [3] and also theoretically predicted by Koning et al. [26] and Glensk et al. [27]. 


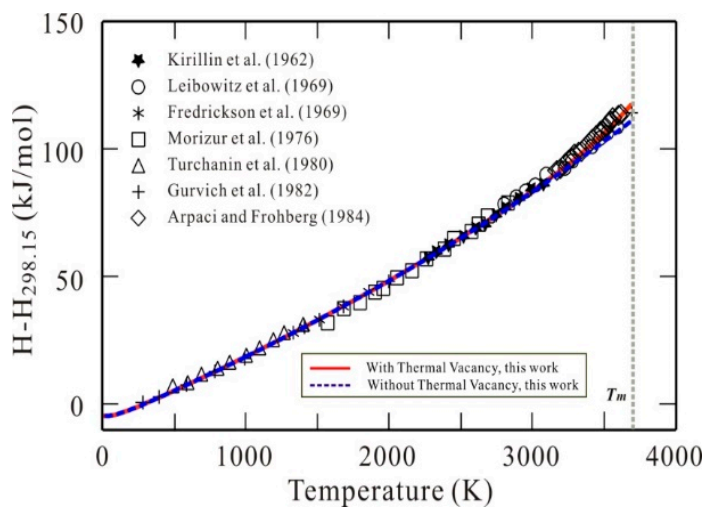

Figure 2. Heat contents $\left(H-H_{298.15}\right)$ of bcc W as a function of temperature. Symbols: Experimental data [14,34-39]. Solid line (red): Calculated results according to the presently established Gibbs energy for bcc W with thermal vacancy contribution. Dashed line (blue): Calculated results according to the presently established Gibbs energy for defect-free bcc W without thermal vacancy contribution.

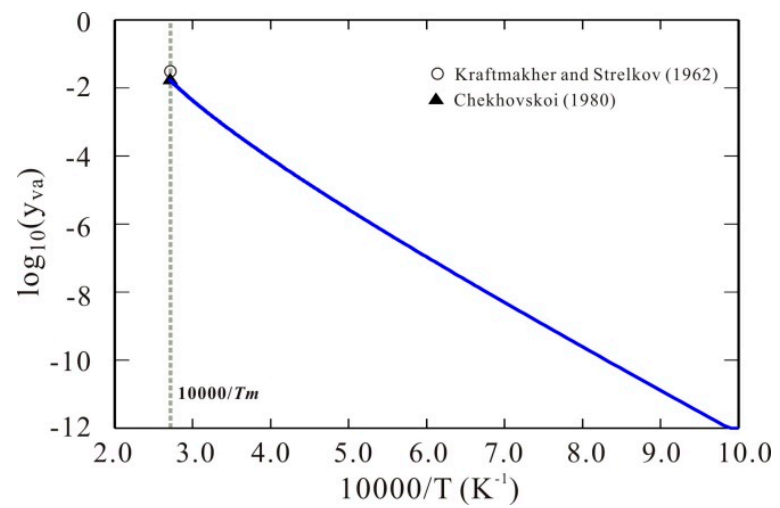

Figure 3. Arrhenius plots of thermal vacancy concentration of bcc W. Symbols: Experimental data at melting point from $[9,10]$. Solid line: Calculated thermal vacancy concentration of bcc W according to the presently established Gibbs energy for bcc W. A clear curvature is obtained for the Arrhenius plot of thermal vacancy concentration of bcc $W$.

\section{Conclusions}

In this paper, a thorough thermodynamic study on the effect of thermal vacancy on strongly non-linear thermodynamic behaviors in bcc W close to its melting point was performed. By considering the contribution of thermal vacancy, a thermodynamic model was presented for describing the Gibbs energy of pure bcc $\mathrm{W}$ from $0 \mathrm{~K}$ to melting point. A new formula for interaction part as well as a pragmatic two-step optimization strategy was proposed. With the obtained Gibbs energy of pure bcc $\mathrm{W}$ assessed from the experimental/first-principles computed thermodynamic quantities, the thermodynamic behaviors in bcc W, including the strongly nonlinear temperature-dependence heat capacity close to the melting point, and a nonlinear Arrhenius plot of vacancy concentration were well reproduced. It is anticipated that the presently proposed thermodynamic model and optimization strategy for bcc W should be universal ones and are applicable to all other metals.

Author Contributions: Both authors Y.T. and L.Z. contributed to the theoretical framework and the interpretation of the results as well as to the preparation of this manuscript.

Funding: This research was funded by National Key Research and Development Program of China (Grant No. 2016YFB0301101) and Hebei Provincial Science and Technology Program of China (Grant No. BJ2018026)—Outstanding Young Talents Plan. 
Acknowledgments: Y. Tang acknowledges the financial support from Yuanguang fellowship released by Hebei University of Technology. L. Zhang acknowledges project supported by the State Key Laboratory of Powder Metallurgy Foundation, Central South University, Changsha, China.

Conflicts of Interest: The authors declare no conflict of interest.

\section{References}

1. Ven, A.V.; Yu, H.C.; Ceder, G.; Thornton, K. Vacancy mediated substitutional diffusion in binary crystalline solids. Prog. Mater. Sci. 2010, 55, 61-105.

2. Protik, N.H.; Carrete, J.; Katcho, N.A.; Mingo, N.; Broido, D. Ab initio study of the effect of vacancies on the thermal conductivity of boron arsenide. Phys. Rev. B Condens. Matter 2016, 94, 045207. [CrossRef]

3. Kraftmakher, Y. Equilibrium vacancies and thermophysical properties of metals. Phys. Rep. 1998, $299,79-188$. [CrossRef]

4. Causey, R.A.; Venhaus, T.J. The use of tungsten in fusion reactors: A review of the hydrogen retention and migration properties. Phys. Scr. 2001, T94, 9-15. [CrossRef]

5. Abernethy, R.G. Predicting the performance of tungsten in a fusion environment: A literature review. Mater. Sci. Technol. 2017, 33, 388-399. [CrossRef]

6. Chekhovskoi, V.Y. The enthalpy and heat capacity of tungsten in the temperature range $400-3500 \mathrm{~K}$. Teplofiz. Vys. Temp. 1980, 18, 1191.

7. Kraftmakher, Y.A.; Strelkov, P.G. Energy of formation and concentration of vacancies in tungsten. Phys. Solid State 1962, 4, 1662-1664.

8. Worthing, A.G. Atomic heats of tungsten and of carbon at incandescent temperatures. Phys. Rev. 1918, 12, 199-225. [CrossRef]

9. Jaeger, F.M.; Rosenbohm, E. The exact measurement of the specific heats of solid substances at high temperatures. III. The specific heats of palladium and of tungsten. Konicki. Ned. Akad. Wetensch. Proc. Ser. B 1930, 33, 457-472.

10. Hoch, M.; Johnston, H.L. A high temperature drop calorimeter: The heat capacities of tantalum and tungsten between 1000 and 3000 K. J. Chem. Phys. 1961, 65, 855-860. [CrossRef]

11. Kirillin, V.A.; Sheindlin, A.E.; Chekhovskoi, V.Y.; Petrov, V.A. Thermodynamic properties of tungsten. Russ. J. Phys. Chem. 1961, 37, 1212.

12. Lowenthal, G.C. The specific heat of metals between $1200 \mathrm{~K}$ and $2400 \mathrm{~K}$. Aust. J. Phys. 1963, 16, 47-67.

13. Hein, R.A.; Flagella, P.N. Enthalpy Measurements of UO2 and Tungsten to 3260 K; GE (General Electric) Report GEMP-578; General Electric: Boston, MA, USA, February 1968.

14. Leibowitz, L.; Chasanov, M.G.; Mishler, L.W. The enthalpy of solid tungsten from $2800 \mathrm{~K}$ to its melting point. Trans. Metall. AIME 1969, 245, 981-984.

15. Yakunkin, M.M. Specific-heat of tungsten by periodic pulsed heating. High Temp. 1983, 21, 848-853.

16. Senchenko, V.N.; Sheindlin, M.A. Experimental investigation of the caloric properties of tungsten and graphite near their melting-points. High Temp. 1987, 25, 364-368.

17. Righini, F.; Spisiak, J.; Bussolino, G.C.; Rosso, A.; Haidar, J. Measurement of thermophysical properties by a pulse-heating method: Thoriated tungsten in the range 1200 to $3600 \mathrm{~K}$. Int. J. Thermophys. 1994, 15, 1311-1322. [CrossRef]

18. Cezairliyan, A.; McClure, J.L. High-speed electrical (subsecond) measurement of heat capacity, resistivity, and thermal radiation properties of tungsten in the range 2000 to 3600 K. J. Res. Nat. Stand. Sec. A 1971, 75, 283-290. [CrossRef]

19. Magnus, A.; Holzmann, H. Untersuchungen über die Spezifischen Wärme von Tantal, Wolfram und Beryllium zwischen 100 und $900{ }^{\circ} \mathrm{C}$. Ann. Phys. 1929, 3, 585-612. [CrossRef]

20. Jaeger, F.M.; Rosenbohm, E. La détermination exacte des chaleurs spécifiques vraies du tungstèn, du rhodium, du palladium, du ruthénium, delosmiumet de l'osmium et l'iridium à des temperatures entre 0 et $1625^{\circ} \mathrm{C}$. Recl. Trav. Chim. Pays-Bas 1932, 51,1-46. [CrossRef]

21. Waiters, T.R.; Craig, R.S.; Wallace, W.E. Heat Capacity of Tungsten between 4 and 15 K. Phys. Rev. 1956, 104, 1240-1241.

22. DeSorbo, W. Low temperature heat capacity of bismuth and tungsten. J. Phys. Chem. 1958, 62, 965-967. [CrossRef] 
23. Clusius, K.; Franzosini, P. Ergebnisse der Tieftemperaturforschung-XXIII Atom- und Elektronenwärme des Molybdäns und Wolframs zwischen $10^{\circ} \mathrm{K}$ und $273^{\circ} \mathrm{K}$. Z. Naturforsch. Teil A 1959, 149, 99-105.

24. Williams, M.E. Ab Initio Elastic and Thermodynamic Properties of High-Temperature Cubic Intermetallics at Finite. Master Thesis, Texas A\&M University, College Station, TX, USA, May 2008.

25. Mundy, J.N.; Rothman, S.J.; Lam, N.Q.; Hoff, H.A.; Nowicki, L.J. Self-diffusion in tungsten. Phys. Rev. B Condens. Matter 1978, 18, 6566. [CrossRef]

26. Koning, M.; Debiaggi, S.R.; Monti, A.M. Vacancy-formation thermodynamics in aluminium and nickel: A computational study. Defect Diffus. Forum 2004, 224, 59-74. [CrossRef]

27. Glensk, A.; Grabowski, B.; Hickel, T.; Neugebauer, J. Breakdown of the Arrhenius law in describing vacancy formation energies: The importance of local anharmonicity revealed by Ab initio thermodynamics. Phys. Rev. X 2014, 4, 011018.

28. Franke, P. Modeling of thermal vacancies in metals within the framework of the compound energy formalism. J. Phase Equilib. Diffus. 2014, 35, 780-787. [CrossRef]

29. Hillert, M. The compound energy formalism. J. Alloys Compd. 2001, 35, 161-176. [CrossRef]

30. Chase, M.W.; Ansara, I.; Dinsdale, A.; Eriksson, G.; Grimvall, G.; Hoglund, L.; Yokokawa, H. Thermodynamic models and data for pure elements and others endmembers of solutions. Calphad 1995, 19, 437-447.

31. Born, M.; Huang, K. Dynamical Theory of Crystal Lattices; Oxford University Press: Oxford, UK, 1954.

32. Grimvall, G. Thermophysical Properties of Materials; Elsevier Science: Amsterdam, The Netherland, 1986.

33. Dinsdale, A.T.; Khvan, A.V.; Watson, A. Critical assessment 5: Thermodynamic data for vacancies. Mater. Sci. Technol. 2014, 30, 1715-1718. [CrossRef]

34. Kirillin, V.A.; Sheindlin, A.E.; Chekhovskoi, V.Y. Enthalpy and specific heat of tungsten in the $0-2400{ }^{\circ} \mathrm{C}$ temperature range. Dokl. Akad. Nauk SSSR 1962, 142, 1323-1326.

35. Fredrickson, D.R.; Kleb, R.; Nuttall, R.L.; Hubbard, W.N. A drop calorimeter with an electron beam heated furnace. Rev. Sci. Instrum. 1969, 40, 1022-1025. [CrossRef]

36. Morizur, G.; Radenac, A.; Cretenet, J.C. Drop calorimetry at 3000 K. High Temp. High Press. 1976, 8, 113-120.

37. Turchanin, A.G.; Babenko, S.A.; Ekimov, S.E. Device for exact determination of solids enthalpy at high temperatures. Tungsten enthalpy in 400-1400 K range. Teplofizika Vysokikh Temperatur 1980, 18, 995-1001.

38. Gurvich, L.V.; Veits, I.V.; Medvedev, V.A. Calculations of Thermodynamic Properties; Nauka: Moscow, 1982.

39. Arpaci, E.; Frohberg, M.G. Enthalpy measurements on solid and liquid tungsten by levitation calorimetry. Z. Metallkd. 1984, 75, 614-618.

40. Walford, L.K. The X-ray debye temperature of tungsten. Mater. Res. Bull. 1969, 4, 137-142. [CrossRef]

41. Cacciamani, G.; Chang, Y.A.; Grimvall, G.; Franke, P.; Kaufman, L.; Miodownik, P.; Sanchez, J.M.; Schalin, M.; Sigli, C. Thermodynamic modelling of solutions and alloys. Group 3: Order-disorder phase diagrams. Calphad 1997, 21, 219-246.

42. Wang, Y.; Curtarolo, S.; Jiang, C.; Arroyave, R.; Wang, T.; Cederc, G.; Chen, L.; Liu, Z. Ab initio lattice stability in comparison with CALPHAD lattice stability. Calphad 2004, 28, 79-90. [CrossRef]

(C) 2018 by the authors. Licensee MDPI, Basel, Switzerland. This article is an open access article distributed under the terms and conditions of the Creative Commons Attribution (CC BY) license (http://creativecommons.org/licenses/by/4.0/). 